\title{
Improved spectrometer-microscope for quantitative fluorescence resonance energy transfer measurement based on simultaneous spectral unmixing of excitation and emission spectra
}

Fangrui Lin

Mengyan Du

Fangfang Yang

Lichun Wei

Tongsheng Chen 


\title{
Improved spectrometer-microscope for quantitative fluorescence resonance energy transfer measurement based on simultaneous spectral unmixing of excitation and emission spectra
}

\author{
Fangrui Lin, Mengyan Du, Fangfang Yang, Lichun Wei, and Tongsheng Chen* \\ South China Normal University, MOE Key Laboratory of Laser Life Science and College of Life Science, Guangzhou, China
}

\begin{abstract}
Based on our recently developed quantitative fluorescence resonance energy transfer (FRET) measurement method using simultaneous spectral unmixing of excitation and emission spectra (ExEm-spFRET), we here set up an improved spectrometer-microscope (SM) for implementing modified ExEm-spFRET (mExEm-spFRET), in which a system correction factor $\left(f_{\mathrm{sc}}\right)$ is introduced. Our SM system is very stable for at least six months. Implementation of mExEm-spFRET with four or two excitation wavelengths on SM for single living cells expressing different FRET constructs obtained consistent FRET efficiency $(E)$ and acceptor-donor concentration ratio $\left(R_{c}\right)$ values. We also performed mExEm-spFRET measurement for single living cells coexpressing cyan fluorescent protein (CFP)-Bax and yellow fluorescent protein (YFP)-Bax and found that the $E$ values between CFP-Bax and YFP-Bax were very low (2.2\%) and independent of $R_{c}$ for control cells, indicating that Bax did not exist as homooligomer in healthy cells, but positively proportional to $R_{c}$ in the case of $R_{c}<1$ and kept constant value (25\%) when $R_{c}>1$ for staurosporine (STS)-treated cells, demonstrating that all Bax formed homooligomer after STS treatment for 6 h. @ 2018 Society of Photo-Optical Instrumentation Engineers (SPIE) [DOI: 10.1117/1. JBO.23.1.016006]
\end{abstract}

Keywords: fluorescence resonance energy transfer; excitation-emission spectral unmixing; spectroscopy; fluorescence microscopy; single living cells.

Paper 170169R received Mar. 18, 2017; accepted for publication Dec. 7, 2017; published online Jan. 8, 2018.

\section{Introduction}

Fluorescence resonance energy transfer (FRET) is an indispensable tool for monitoring intracellular instantaneous and weak biological processes in real time, including protein-protein interaction, ${ }^{1}$ conformational changes of proteins, ${ }^{2}$ activation of proteins kinases, ${ }^{3-6}$ and dynamic concentration changes of ions. ${ }^{7,8}$ Quantitative FRET signals, including FRET efficiency $(E)$ and the concentration ratio $\left(R_{c}\right)$ between acceptor and donor molecules, are essential for scientific communication and exact interpretation. ${ }^{9-11}$ However, the prerequisite of larger overlap between donor emission spectra and acceptor excitation spectra for FRET occurrence inevitably results in a significant overlap between donor and acceptor emission spectra (named as donor emission cross talk). ${ }^{12}$ Moreover, acceptor can also be excited directly under the excitation wavelengths of donor fluorophores (named as acceptor excitation cross talk). ${ }^{13-16}$ The two spectral cross talks preclude separation of three spectral components: donor fluorescence, direct excitation acceptor fluorescence, and FRET-sensitized acceptor fluorescence. ${ }^{17}$

In 1992, Clegg ${ }^{18}$ described the concept of unmixing fluorescence spectra to gain FRET efficiency. Spectral linear unmixing of emission spectra (Em unmixing) has been widely used for quantitative FRET measurement. ${ }^{14,15,19,20}$ Contributions of donor and acceptor to the emission spectra of a given FRET pair can be easily resolved by Em unmixing due to their different emission spectra. However, the acceptor excitation cross talk

*Address all correspondence to: Tongsheng Chen, E-mail: chentsh126@126 .com must be corrected using an additional acceptor reference because of the same spectra of direct acceptor emission and FRET-sensitized acceptor emission. ${ }^{13-15,17,21}$ The concept used for Em unmixing can also be applied to spectral unmixing of excitation spectra (Ex unmixing). ${ }^{22}$ Moreover, spectral linear unmixing of the combined excitation and emission spectra (ExEm unmixing) has the inherent ability to resolve the donor fluorescence, direct excitation acceptor fluorescence, and FRET-sensitized acceptor fluorescence without additional reference. ${ }^{16,17,22}$

With the advances of fluorescence spectroscopy and microscopy, ExEm unmixing has been tried for quantitative FRET measurement (ExEm-spFRET method). ${ }^{17,23}$ In 2013, Mustafa et al. ${ }^{16}$ demonstrated that ExEm-spFRET measurement with as few as two excitation wavelengths could obtain accurate $E$ values and performed ExEm-spFRET measurement on a laser scanning confocal microscope with 405- and 488-nm excitation wavelengths for single living cells expressing a fluorescent Cerulean-Venus tandem construct. We recently set up a spectrometer-microscope (SM) by combining a fiber optic spectrometer and a wide-field fluorescence microscope for fast and high-sensitive quantitative FRET measurement using Em unmixing ${ }^{24}$ and also developed a wide-field microscope equipped with a liquid crystal tunable filter for quantitative ExEm-spFRET imaging in single living cells. ${ }^{25}$

In this report, we improved the SM system for implementing quantitative ExEm-spFRET measurement in single living

$1083-3668 / 2018 / \$ 25.00$ @ 2018 SPIE 


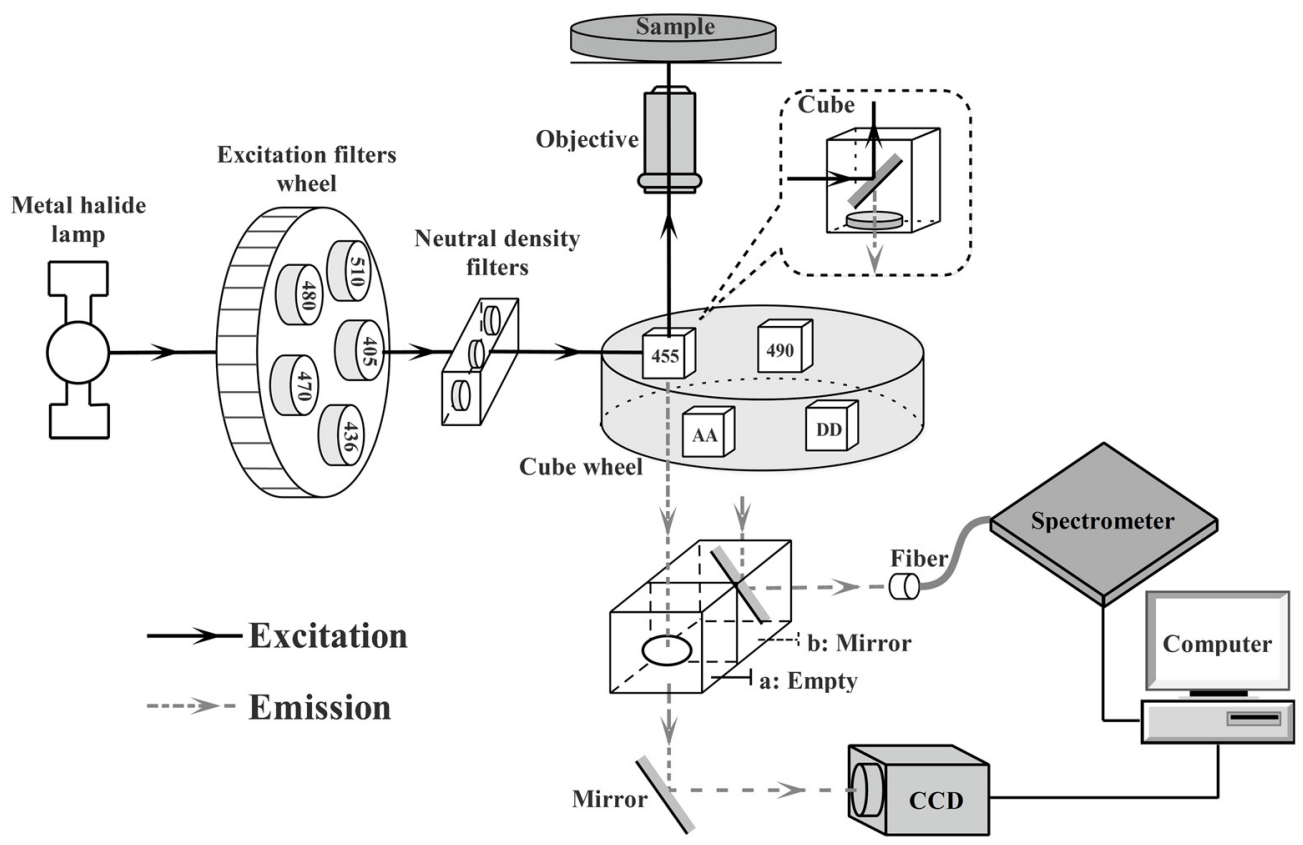

Fig. 1 Illustration of SM. Excitation filters wheel contains five bandpass excitation filters: Ex405, Ex436, Ex470, Ex480, and Ex510. Cube wheel contains four cubes: D455 cube containing a 455-nm dichroic mirror (D455) and a long-pass emission filter of $460 \mathrm{~nm}$ (LP460), D490 cube containing a 490-nm dichroic mirror (D490) and a long-pass emission filter of $495 \mathrm{~nm}$ (LP495), DD cube containing a 460-nm dichroic mirror (D460) and a bandpass emission filter of $480 / 30 \mathrm{~nm}$ (BP480/30), and AA cube containing a 515-nm dichroic mirror (D515) and a bandpass emission filter of 550/40 nm (BP550/40). Two detection channels: CCD imaging channel (a) and spectral detection channel (b).

cells. Moreover, a system correction factor $\left(f_{\mathrm{sc}}\right)$ that can be predetermined using a donor-acceptor tandem reference with known FRET efficiency is introduced to modify ExEmspFRET method (mExEm-spFRET). mExEm-spFRET not only has the inherent ability of resolving the donor emission cross talk and acceptor excitation cross talk without additional reference but also eliminates the influence of the emission transmission characteristics of the instrument used on quantitative FRET measurement. We implemented mExEm-spFRET measurement with four $(405,436,470$, and $480 \mathrm{~nm})$ or two (405 and $470 \mathrm{~nm}$, 405 and $480 \mathrm{~nm}, 436$ and $470 \mathrm{~nm}$, and 436 and $480 \mathrm{~nm}$ ) excitation wavelengths on our SM platform for single living cells expressing different FRET constructs and obtained consistent $E$ and $R_{c}$ values. Quantitative mExEm-spFRET measurement for HeLa cells coexpressing CFP-Bax and YFP-Bax showed that the $E$ values between CFP-Bax and YFP-Bax were about $2.2 \%$ independent of $R_{c}$ for control cells, indicating that Bax did not exist as homooligomer in healthy cells, but positively proportional to $R_{c}$ in the case of $R_{c}<1$ and kept constant value (25\%) when $R_{c}>1$ for staurosporine (STS)-treated cells, demonstrating that all Bax formed homooligomer after STS treatment for $6 \mathrm{~h}$.

\section{Materials and Methods}

\subsection{Improved Spectrometer-Microscope}

The improved SM consists of a wide-field fluorescence microscope (IX73, Olympus, Japan) equipped with a metal halide lamp (HGLGPS, Olympus, Japan), a $40 \times / 1.3$ NA oil objective (UPLFLN40XO, Olympus, Japan), a CCD (ORCA-Flash 4.0, Hamamatsu, Japan), a fiber optic spectrometer (QE65 Pro, Ocean Optics, Florida), and a customized excitation filters wheel. As shown in Fig. 1, five different bandpass excitation filters are installed in the excitation filters wheel. In our study, four different excitations of 405/20 nm (Ex405), 436/20 nm (Ex436), 470/20 nm (Ex470), and 480/20 nm (Ex480) (Chroma, United States) are used for ExEm-spFRET and mExEm-spFRET measurement. Excitation of 510/20 nm (Ex510) (Chroma, United States) is used for emp-PbFRET measurement. Excitations of both Ex405 and Ex436 share the same filter cube that contains a DM455 (455-nm dichroic mirror, D455) (Olympus, Japan) and an ET460lp (long-pass emission filter of $460 \mathrm{~nm}$, LP460) (Chroma, United States). Similarly, excitations of both Ex470 and Ex480 share another filter cube that contains a DM490 (490-nm dichroic mirror, D490) (Olympus, Japan) and an ET495lp (longpass emission filter of $495 \mathrm{~nm}, \mathrm{LP} 495)$ (Chroma, United States). The illumination intensity can be attenuated in seven discrete steps $(0 \%, 3 \%, 6 \%, 12 \%, 25 \%, 50 \%$, and $100 \%)$, and another neutral density filters controller with three discrete steps $(1 \%$, $3 \%$, and empty) can be used for the same purpose. Donor excitation and donor detection (DD) cube containing a DM460 (460$\mathrm{nm}$ dichroic mirror, D460) and an ET480/30m (bandpass emission filter of 480/30 nm, BP480/30) (Chroma, United States) and acceptor excitation and acceptor detection (AA) cube containing a DM515 (515-nm dichroic mirror, D515) and an ET550/40m (bandpass emission filter of 550/40 nm, BP550/40) (Chroma, United States) are used to estimate the coexpression of both donor and acceptor in single living cells.

SM has two independent detection modes: (a) microscopic imaging in CCD channel, offering a guidance for finding cells, and (b) spectral detection in spectrometer channel, recording emission spectra of the guided cells in the middle of CCD channel. Each $\operatorname{count}(\lambda)$ or $E(\lambda)$ at the emission wavelength is related to the photons in about a $0.761-\mathrm{nm}$ wavelength range. Spectral detection range is from 460 to $620 \mathrm{~nm}$ in this report. 


\subsection{Modified ExEm-spFRET Method}

ExEm-spFRET method we recently developed ${ }^{25}$ is modified using a system correction factor $\left(f_{\mathrm{sc}}\right)$ as follows (mExEmspFRET):

$E=\frac{W_{S}}{f_{\mathrm{sc}} r_{Q} W_{D}+W_{S}}$,

$R_{c}=\frac{W_{A}}{r_{k}\left(f_{\mathrm{sc}} r_{Q} W_{D}+W_{S}\right)}$

where $r_{Q}$ is the quantum yield ratio of acceptor to donor, $r_{k}$ is defined as the ratio of total acceptor extinction coefficient to total donor extinction coefficient at all excitation wavelengths, and $W_{D}, W_{A}$, and $W_{S}$ are the weight factors of donor, acceptor, and donor-acceptor sensitization, respectively. Linearly unmixing the measured excitation-emission spectrum $\left(S_{D A}\right)$ of an FRET sample into the unit-area-normalized excitation-emission spectral fingerprints of donor $\left(S_{D}\right)$ and acceptor $\left(S_{A}\right)$ as well as donor-acceptor sensitization $\left(S_{S}\right)$ is as follows: ${ }^{25}$

$S_{D A}=W_{D} \cdot S_{D}+W_{A} \cdot S_{A}+W_{S} \cdot S_{S}$

A donor-acceptor tandem reference with known $E^{\text {ref }}$ and $R_{C}^{\text {ref }}$ can be used to predetermine $f_{\mathrm{sc}}$ and $r_{k}$ as follows:

$f_{\mathrm{sc}}=\frac{W_{S}-W_{S} E^{\mathrm{ref}}}{r_{Q} W_{D} E^{\mathrm{ref}}}$,

$r_{k}=\frac{W_{A}}{R_{C}^{\mathrm{ref}}\left(f_{\mathrm{sc}} r_{Q} W_{D}+W_{S}\right)}$.

In reality, $E^{\text {ref }}$ can also be determined using some FRET methods, such as three-cube-based acceptor-sensitized emission method (E-FRET) ${ }^{10}$ or partial acceptor photobleaching method (emp-PbFRET). ${ }^{26}$

\subsection{Partial Acceptor Photobleaching Method}

Quantitative emp-PbFRET measurement was performed on SM for predetermining the $E^{\text {ref }}$ of a 1D-nA tandem reference which contains one donor $(D)$ and $n$ acceptor $(A)$. Ex436 excitation was used to excite donor (Cerulean/CFP), and Ex510 excitation was used to selectively excite acceptor (Venus/YFP). Donor detection channel $\left(\mathrm{CH}_{D}\right)$ from 470 to $490 \mathrm{~nm}$ was used to selectively collect donor emission, and acceptor detection channel $\left(\mathrm{CH}_{A}\right)$ from 530 to $550 \mathrm{~nm}$ was used to mainly collect acceptor emission. The $E^{\text {ref }}$ value of $1 \mathrm{D}-\mathrm{nA}$ construct can be measured as follows: $:^{1,26}$

$E=\frac{1-\frac{I_{\mathrm{DD}}}{I_{\mathrm{DD}}^{\text {post }}}}{1-\frac{I_{\mathrm{DD}}}{I_{\mathrm{DD}}^{\text {post }}}(1-x)}$,

where $I_{\mathrm{DD}}$ and $I_{\mathrm{DD}}^{\mathrm{post}}$ are the donor intensity (fluorescence count) in $\mathrm{CH}_{D}$ channel with donor excitation before and after partial acceptor photobleaching, respectively. $I_{\mathrm{AA}}$ and $I_{\mathrm{AA}}^{\text {post }}$ are the acceptor intensity (fluorescence count) in $\mathrm{CH}_{A}$ channel with selective acceptor excitation before and after partial acceptor photobleaching, respectively, and $x$ is the photobleaching degree of acceptor calculated as $x=\left(I_{\mathrm{AA}}-I_{\mathrm{AA}}^{\mathrm{post}}\right) / I_{\mathrm{AA}}$.

\subsection{Calibration of SM}

Careful calibration of SM was carried out with a halogen tungsten lamp (ISP-REF-CAL, Ocean Optics, Dunedin, Florida) just as described previously. ${ }^{24}$ We first used a spectrometer (QE65 Pro, Ocean Optics, Florida) precalibrated by a standard light source (LS-1-CAL, Ocean Optics, Florida) to measure the spectrum $[E(\lambda)]$ of the halogen tungsten lamp. We next used the spectrometer to measure the spectrum [count lamp $(\lambda)$ ] at the export of our microscope when the halogen tungsten lamp was placed on the objective of the microscope. The emission spectral response was calculated using $K(\lambda)=$ $E(\lambda) /$ count $_{\text {lamp }}(\lambda)$.

\subsection{Reagent and Plasmids}

Plasmids DNA of Cerulean (C), Venus (V), CFP, and YFP were purchased from Addgene Company (Cambridge, Massachusetts). FRET tandem constructs, including $\mathrm{C} 32 \mathrm{~V}$, CVC, and VCV, were kindly provided by the Vogel lab (National Institutes of Health, Bethesda, Maryland). ${ }^{19,27}$ Plasmids DNA of CFP-Bax and YFP-Bax were kindly provided by Dr. Prehn. ${ }^{1}$ Plasmid DNA of 18AA was kindly given by Professor Kaminski. ${ }^{11}$ STS was purchased from Sigma-Aldrich Co. LLC (Santa Clara).

\subsection{Cell Culture and Transfection}

HeLa cells obtained from the Department of Medicine, Jinan University (Guangzhou, China) were cultured just as described previously. ${ }^{28}$ When the cells reached $70 \%$ to $90 \%$ confluence in a $35-\mathrm{mm}$ glass dish, plasmids were transfected into cells by Turbofect $^{\mathrm{TM}}$ (Fermentas Inc., Glen Burnie, Maryland) for $24 \mathrm{~h}$.

\section{Results and Discussion}

\subsection{Calibration of SM}

We first measured the emission spectral responses $[K(\lambda)]$ of our SM system as shown in Fig. 7. We used the spectrometer to measure the spectrum $[E(\lambda)$, black solid line] of the halogen tungsten lamp and the spectrum [count $\mathrm{lamp}(\lambda)$, black dot line] at the export of our microscope with D455 cube (a) and D490 cube (b), respectively, when the halogen tungsten lamp was placed on the objective of our microscope. $K_{1}(\lambda)$ for D455 cube and $K_{2}(\lambda)$ for D490 cube are also shown in Fig. 7 (gray solid line). Throughout the paper, emission count spectra with Ex405 or Ex436 excitation were calibrated with $K_{1}(\lambda)$, and emission count spectra with Ex470 or Ex480 excitation were calibrated with $K_{2}(\lambda)$.

We found that the emission spectral responses measured during six months were constant, demonstrating the excellent stability of our SM system. Although this calibration step is not mandatory for a precalibrated SM during at least six months, we actually performed this calibration step for every mExEmspFRET measurement, which can be used as a criterion to determine whether SM is stable. In fact, this calibration step is very simple and can be performed within a few minutes. 

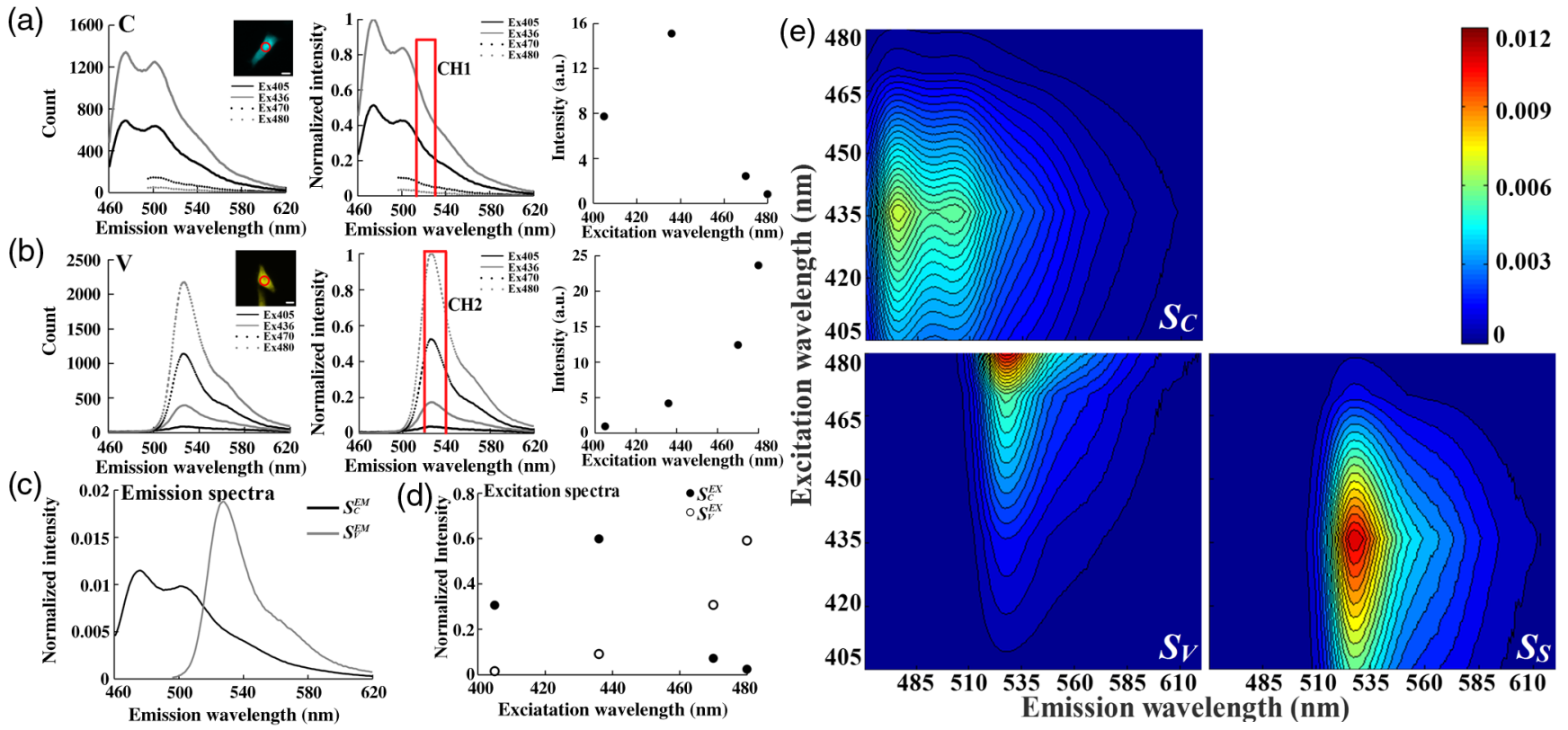

Fig. 2 Excitation-emission spectral fingerprints of Cerulean and Venus as well as Cerulean-Venus sensitization. (a) Raw count spectra (left), normalized intensity spectra after calibration (middle), and the relative fluorescence intensity (right) in emission channel $\mathrm{CH} 1$ (from 510 to $530 \mathrm{~nm}$ ) of a representative HeLa cell expressing Cerulean-only (inset image) with Ex405, Ex436, Ex470, and Ex480 excitation, respectively, with respect to the value of the maximum peak at emission spectrum with Ex436 excitation. Scale bar: $10 \mu \mathrm{m}$; (b) raw count spectra (left), normalized intensity spectra after calibration (middle), and the relative fluorescence intensity (right) in emission channel $\mathrm{CH} 2$ (from 520 to $540 \mathrm{~nm}$ ) of a representative HeLa cell expressing Venus-only (inset image) with Ex405, Ex436, Ex470, and Ex480 excitation, respectively, with respect to the value of the maximum peak at emission spectrum with Ex436 excitation. Scale bar: $10 \mu \mathrm{m}$; (c) unit-area-normalized emission spectra of Cerulean (SEM C) and Venus (SEM V) measured from at least 20 living HeLa cells; (d) unit-area-normalized excitation spectra of Cerulean (SEX C) and Venus (SEX V) measured from at least 20 living HeLa cells; (e) three excitation-emission spectral fingerprints of Cerulean $\left(S_{C}\right)$, Venus $\left(S_{V}\right)$, and Cerulean-Venus sensitization $\left(S_{S}\right)$ obtained by the outer product of the excitation and emission spectra in (c) and (d).

\subsection{Excitation-Emission Spectral Fingerprints}

Living HeLa cells exclusively expressed Cerulean (donor) or Venus (acceptor) were used to measure the excitation-emission spectral fingerprints of Cerulean and Venus as well as CeruleanVenus sensitization on SM. Mean background spectrum with four excitations, respectively, collected from 20 nontransfected (no DNA plasmids and vectors) cells was subtracted from the raw count spectrum from cells expressing fluorescent proteins. Figure 2(a) (left) shows the raw emission count spectra of a representative cell expressing Cerulean-only indicated by red circle (inset) with Ex405, Ex436, Ex470, and Ex480 excitation, respectively. After calibration with $K(\lambda)$, emission spectrum of Cerulean with four excitations, respectively, was divided by the maximum value of emission spectrum with Ex436 excitation to obtain the normalized emission spectra [Fig. 2(a), middle]. The relative fluorescence intensities in emission wavelength range of 510 to $530 \mathrm{~nm}(\mathrm{CH} 1)$ with Ex405, Ex436, Ex470, and Ex480 excitation, respectively, are shown in Fig. 2(a) (right). Similarly, Fig. 2(b) (left) shows the raw emission count spectra of a representative cell expressing Venus-only indicated by red circle (inset) with four excitations. Emission spectrum of Venus with four excitations, respectively, was divided by the maximum value of emission spectrum with Ex480 excitation to obtain the normalized emission spectra [Fig. 2(b), middle]. The relative fluorescence intensities in emission wavelength range of 520 to $540 \mathrm{~nm}(\mathrm{CH} 2)$ with Ex405, Ex436, Ex470, and Ex480 excitation, respectively, are shown in Fig. 2(b) (right). Fluorescence intensities in Fig. 2(a) (middle) with Ex436 excitation and in Fig. 2(b) (middle) with Ex470 excitation are normalized to unit area, respectively, as the emission spectra of Cerulean and Venus.

The emission spectra of Cerulean with Ex436 excitation and Venus with Ex470 excitation obtained from at least 20 living HeLa cells expressing Cerulean or Venus were normalized to unit area as the emission spectra of Cerulean (SEM C) and Venus (SEM V) [Fig. 2(c)]. Figure 2(d) shows the unit-area-normalized excitation spectra of Cerulean (SEX C) and Venus (SEX $\mathrm{V})$. The unit-volume-normalized three-dimensional excitationemission spectral fingerprints of Cerulean $\left(S_{C}\right)$, Venus $\left(S_{V}\right)$, and Cerulean-Venus sensitization $\left(S_{S}\right)$ in Fig. 2(e) were calculated by the outer product of SEX C and SEM C, SEX V and SEM V, and SEX C and SEM V, respectively. In reality, $S_{C}$ and $S_{V}$ as well as $S_{S}$ in Fig. 2(e) were reconstructed by equally dividing the normalized intensity values into 25 grades (pseudocolor), and the equal grades were connected with contours.

The fact that the normalized emission spectra of fluorescent proteins (FPs) (Cerulean/CFP or Venus/YFP) measured from living HeLa or HepG2 cells expressing different levels of FPs are consistent further demonstrates the notion that the absorption and emission spectra of fluorescent proteins are generally very stable. ${ }^{29,30}$ Although fluorescence intensity is proportional to the intensity of excitation light, mExEm-spFRET method is independent of the intensity of excitation light. 
(a)

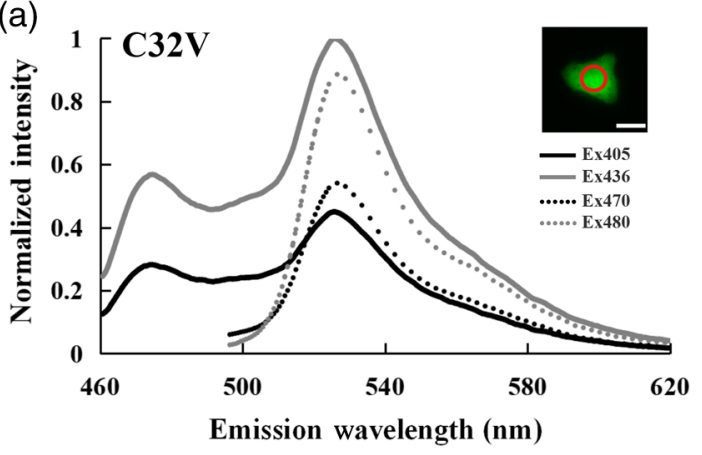

(c)

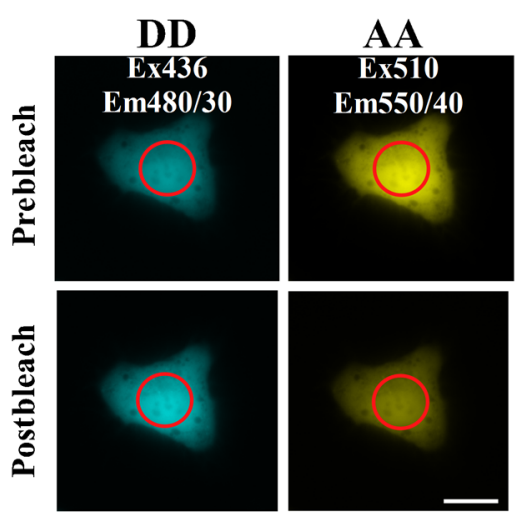

(e)

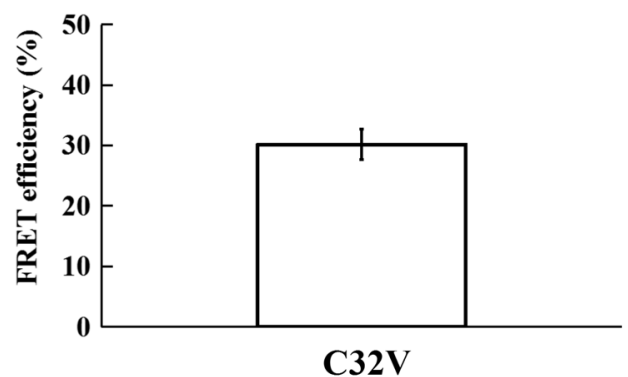

(b)

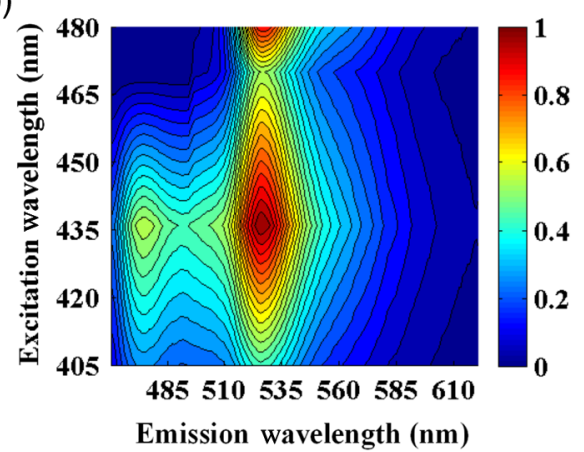

(d)

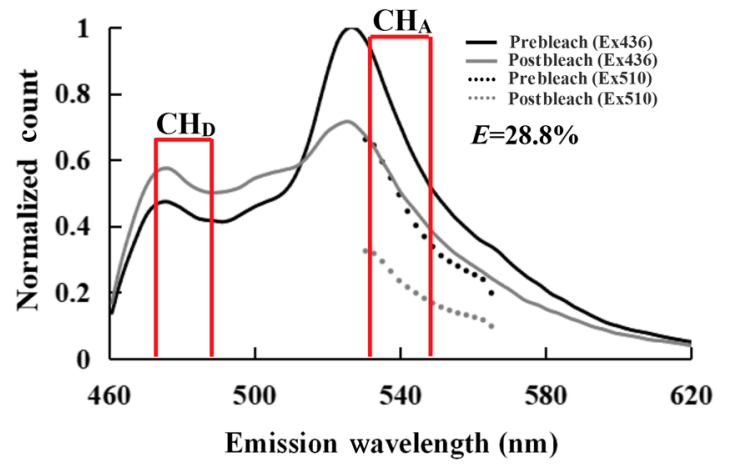

(f)

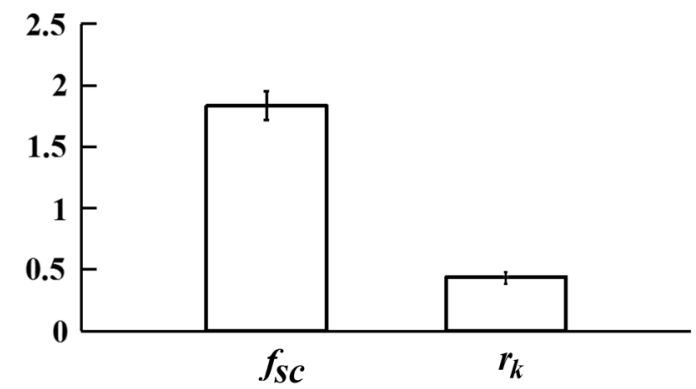

Fig. 3 Predetermination of correction factors $\left(f_{\mathrm{sc}}\right.$ and $\left.r_{k}\right)$ using a Cerulean-Venus tandem construct (C32V). (a) Normalized emission spectra of a representative cell expressing C32V with Ex405, Ex436, Ex470, and Ex480 excitation, respectively, with respect to the value of the maximum peak at emission spectrum with Ex436 excitation. Scale bar: $10 \mu \mathrm{m}$; (b) excitation-emission spectrum corresponding to (a); (c) images of the cell with Ex436 (DD) or Ex510 (AA) excitation before (upper panels) and after (lower panels) partial Venus photobleaching. Scale bar: $10 \mu \mathrm{m}$; (d) normalized count spectra of C32V inside the cell indicated by red circles in (c). $\mathrm{CH}_{\mathrm{D}}: 470$ to $490 \mathrm{~nm}$ and $\mathrm{CH}_{\mathrm{A}}: 530$ to $550 \mathrm{~nm}$. (e) Statistical $E^{\text {ref }}$ value from at least 15 living cells using emp-PbFRET method. (f) Statistical $f_{\mathrm{sc}}$ and $r_{k}$ values from at least 20 living cells.

Because three excitation-emission spectral fingerprints $\left(S_{D}, S_{A}\right.$, and $S_{S}$ ) are normalized to unit volume, different excitation intensity only affects the weight factors $\left(W_{D}, W_{A}\right.$, and $\left.W_{S}\right)$ rather than the ratios of weight factors. In reality, we found that the normalized excitation-emission spectral fingerprints of FPs obtained from living HeLa or HepG2 cells were constant during at least six months, indicating that our SM system is very stable. Therefore, the predetermined $S_{C}, S_{V}$, and $S_{S}$ can be directly used for subsequent quantitative $\mathrm{mExEm}$-spFRET measurement without additional measurement. To offset the random fluctuation of count recorded at different emission wavelength, we summated the fluorescence intensity values in an emission wavelength range ( $\mathrm{CH} 1$ for Cerulean and $\mathrm{CH} 2$ for Venus) rather than at single emission wavelength for Cerulean or Venus to obtain their excitation spectra. In reality, the emission wavelength range of 500 to $530 \mathrm{~nm}$ should be a better choice for $\mathrm{CH} 1$.

\subsection{Predetermination of the Correction Factors $\left(f_{\text {sc }}\right.$ and $\left.r_{k}\right)$}

To predetermine the correction factors $\left(f_{\mathrm{sc}}\right.$ and $\left.r_{k}\right)$, living HeLa cells expressing C32V were excited with Ex405, Ex436, Ex470, and Ex480 excitation, respectively. Figure 3(a) shows the 
(a)

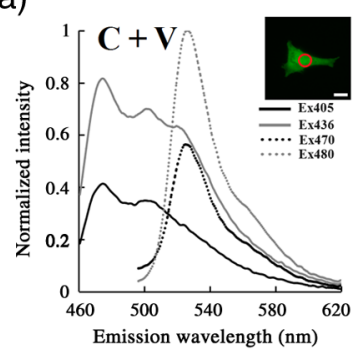

(c)

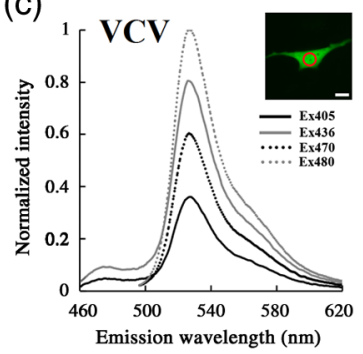

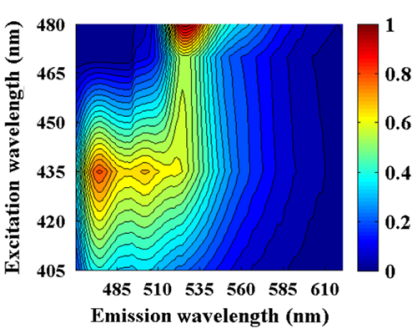

(b)
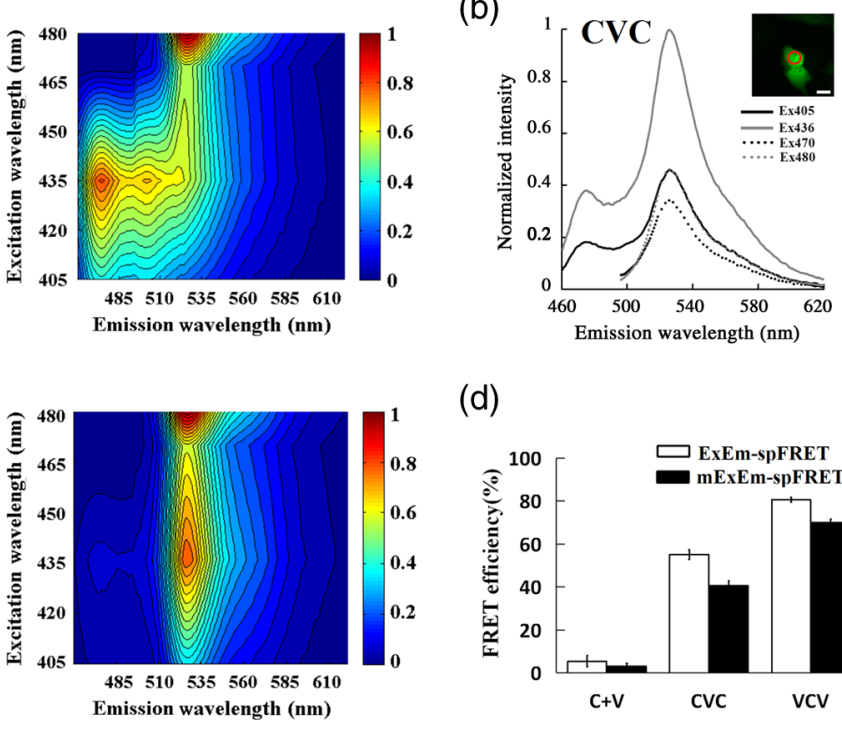

(d)

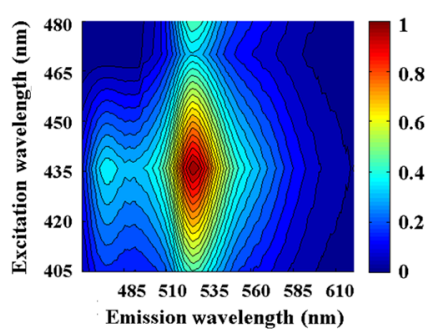

Emission wavelength $(\mathrm{nm})$

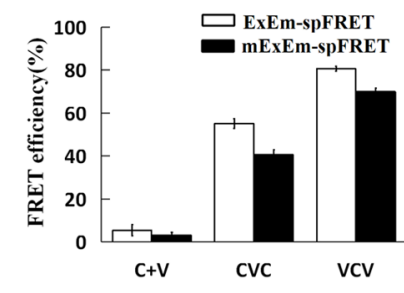

Fig. 4 Implementation of mExEm-spFRET method on SM system for quantitative FRET measurement in living cells separately expressing C + V, CVC, and VCV. (a-C) Normalized emission spectra (left) and corresponding excitation-emission spectrum (right) of representative cells separately expressing $\mathrm{C}+\mathrm{V}$ (a, with respect to the value of the maximum peak at emission spectrum with Ex480 excitation), CVC (b, with respect to the value of the maximum peak at emission spectrum with Ex436 excitation), and VCV (c, with respect to the value of the maximum peak at emission spectrum with Ex480 excitation) with Ex405, Ex436, Ex470, and Ex480 excitation, respectively. Scale bar: $10 \mu \mathrm{m}$. (d) Statistical $E$ (left) and $R_{c}$ (right) values of $\mathrm{C}+\mathrm{V}, \mathrm{CVC}$, and VCV constructs in 20 living HeLa cells obtained by ExEm-spFRET and mExEm-spFRET method, respectively.

normalized emission spectra of a representative cell expressing C32V with Ex405, Ex436, Ex470, and Ex480 excitation, respectively, with respect to the value of the maximum peak at emission spectrum with Ex436 excitation. Figure 3(b) shows the corresponding excitation-emission spectrum $\left(S_{D A}\right)$, which was linearly unmixed according to Eq. (3) to obtain $W_{D}=0.37$, $W_{A}=0.39$, and $W_{S}=0.27$. Next, we used emp-PbFRET method to measure the $E^{\text {ref }}$ value of $\mathrm{C} 32 \mathrm{~V}$ for the same cell. Figure 3(c) shows the fluorescent images of the cell with DD cube (left) and AA cube (right), respectively, before (upper panel) and after (lower panel) partial Venus bleaching. Figure 3(d) shows the corresponding normalized count spectrum with Ex436 (solid line) and Ex510 (dot line) excitation, respectively, before (black) and after (gray) partial Venus bleaching with respect to the maximum value of the count spectrum with Ex436 excitation before partial Venus bleaching (black solid line). We summated the normalized count values in $\mathrm{CH}_{D}$ channel and $\mathrm{CH}_{A}$ channel, respectively, to obtain $I_{\mathrm{DD}}=11.94$, $I_{\mathrm{DD}}^{\mathrm{post}}=14.41, I_{\mathrm{AA}}=13.45$, and $I_{\mathrm{AA}}^{\mathrm{post}}=6.75$. According to Eq. (6), the corresponding $E^{\text {ref }}$ was $28.8 \%$, and the statistical $E^{\text {ref }}$ value measured from 15 living HeLa cells was $30.1 \% \pm$ $2.9 \%$ [Fig. 3(e)]. Substituting $W_{D}=0.37, W_{A}=0.39$, and $W_{S}=0.27$ as well as $E^{\text {ref }}=30.1 \%$ into Eq. (4) to obtain $f_{\mathrm{sc}}=1.81$, where the quantum yield ratio $\left(r_{Q}\right)$ of Venus $(0.57)$ to Cerulean (0.62) is $0.919,,^{31,32}$ and into Eq. (5) to obtain $r_{k}$ was 0.43 , where $R_{C}^{\text {ref }}=1$. Statistical $f_{\mathrm{sc}}$ and $r_{k}$ values from 20 cells are $1.83 \pm 0.12$ and $0.44 \pm 0.01$, respectively [Fig. 3(f)].

In many reports, the quantum yield values of donor $\left(Q_{D}\right)$ and acceptor $\left(Q_{A}\right)$ from literature were directly quoted for quantitative FRET measurement. ${ }^{11,14,20,25,28}$ However, real $Q_{D}$ and $Q_{A}$ values are related to not only the optical properties of donor/ acceptor but also the emission transmission characteristics of the instrument used. Moreover, it is also inappropriate to consider the $Q_{D}$ and $Q_{A}$ values from literature as the real $Q_{D}$ and $Q_{A}$ within a bandpass emission wavelength range. We here used the $f_{\text {sc }}$ to correct the ratio of $Q_{A}$ to $Q_{D}\left(Q_{A} / Q_{D}\right)$ in our SM system. In fact, the product of the $Q_{A} / Q_{D}$ ratio quoted from literature and the $f_{\mathrm{sc}}$ is the real $Q_{A} / Q_{D}$ value in our SM system. Therefore, mExEm-spFRET method can measure the real $Q_{A} / Q_{D}$ value rather than the referenced $Q_{A} / Q_{D}$ value from literature for quantitative FRET measurement.

Generally, $r_{k}$ is only related to the excitation spectrum of our SM system and the absorption spectra of both donor and acceptor for a given cell line. ${ }^{25}$ Just as discussed above about the spectral fingerprints, the spectral characteristics of our SM system and donor/acceptor are very stable. Therefore, for a given specific system, the predetermined $f_{\text {sc }}$ and $r_{k}$ can be directly used for subsequent mExEm-spFRET measurement. In reality, we remeasured $f_{\mathrm{sc}}$ and $r_{k}$ values for CeruleanVenus pair inside HeLa cells on our SM system during six months and obtained consistent $f_{\mathrm{sc}}$ and $r_{k}$ values, further demonstrating the stability of our instrument.

\subsection{Implementation of mExEm-spFRET in Single Living HeLa Cells Expressing $C+V, C V C$, and VCV}

We next performed ExEm-spFRET and mExEm-spFRET method, respectively, on SM to measure the $E$ and $R_{c}$ values of single living cells expressing unlinked Cerulean plus Venus $(\mathrm{C}+\mathrm{V}), \mathrm{CVC}$, and VCV, respectively. We measured four emission spectra of the cells excited with Ex405, Ex436, Ex470, and Ex480, respectively. Figures 4(a)-4(c) show the normalized 
Table 1 Weight values for different constructs.

\begin{tabular}{lccc}
\hline & \multicolumn{3}{c}{ Constructs } \\
\cline { 2 - 4 } Weight values & $\mathrm{C}+\mathrm{V}$ & $\mathrm{CVC}$ & $\mathrm{VCV}$ \\
\hline$W_{D}$ & 0.56 & 0.36 & 0.09 \\
$W_{A}$ & 0.43 & 0.25 & 0.56 \\
$W_{S}$ & 0.03 & 0.41 & 0.34 \\
\hline
\end{tabular}

emission spectra (left) of a representative cell expressing $\mathrm{C}+\mathrm{V}$ (a, with respect to the value of the maximum peak at emission spectrum with Ex480 excitation), CVC (b, with respect to the value of the maximum peak at emission spectrum with Ex436 excitation), and VCV (c, with respect to the value of the maximum peak at emission spectrum with Ex480 excitation), respectively, with different excitations and the corresponding excitation-emission spectra $\left(S_{\mathrm{DA}}\right)$ (right). The $S_{\mathrm{DA}}$ were linearly unmixed according to Eq. (3) to obtain the weight values of donor, acceptor, and donor-acceptor sensitization (Table 1). Substituting these weight values and $r_{k}=0.44$ as well as $f_{\mathrm{sc}}=$ 1.83 into mExEm-spFRET method [Eqs. (1) and (2)] to obtain the corresponding $E$ and $R_{c}: 3.1 \%$ and 1.01 for $\mathrm{C}+\mathrm{V}, 40.4 \%$ and 0.54 for CVC, and $69.2 \%$ and 2.59 for VCV. In addition, for the same cells, implementation of ExEm-spFRET method exhibited that the $E$ and $R_{C}$ values were $5.5 \%$ and 1.21 for $\mathrm{C}+\mathrm{V}, 55.3 \%$ and 0.56 for CVC, and $80.4 \%$ and 2.04 for VCV. Figure 4(d) shows the statistical $E$ and $R_{c}$ values of $\mathrm{C}+\mathrm{V}, \mathrm{CVC}$, and VCV from 20 living cells. The $E$ values of CVC and VCV obtained by mExEm-spFRET are consistent with those measured by E-FRET method $(40.0 \% \pm 0.7 \%$ for CVC and $69.3 \% \pm 1.0 \%$ for VCV). ${ }^{33}$

We also used mExEm-spFRET method with two excitations to calculate the $E$ and $R_{c}$ values of C $+\mathrm{V}, \mathrm{C} 32 \mathrm{~V}, \mathrm{CVC}$, and VCV constructs, respectively, for the same cells (Table 2). mExEmspFRET method with Ex405 and Ex470, Ex405 and Ex480, Ex 430 and Ex470, or Ex436 and Ex480 excitations showed consistent results, while mExEm-spFRET method with Ex405 and Ex436 excitations obtained an obviously larger $R_{c}$ value for $\mathrm{CVC}$ construct, which may owe to the similarity of fluorescence intensity spectra with Ex405 and Ex436 excitation, respectively.

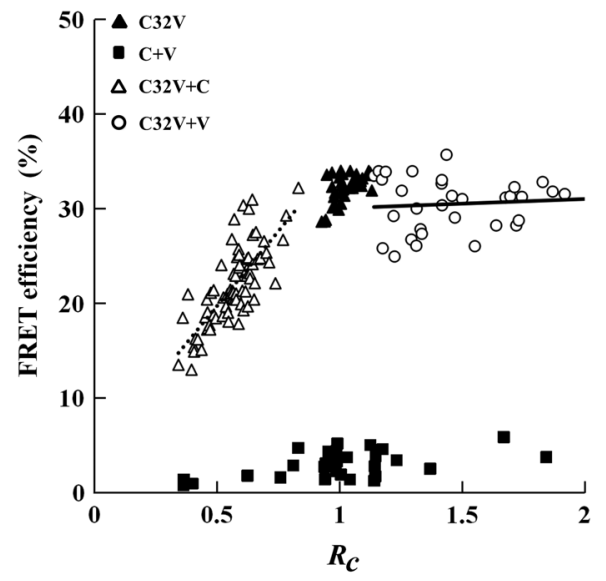

Fig. $5 E$ as a function of $R_{c}$. Implementation of mExEm-spFRET with four excitation wavelengths on SM for the HeLa cells expressing $\mathrm{C} 32 \mathrm{~V}$ (solid triangles), $\mathrm{C}+\mathrm{V}$ (solid squares), C32V + C (open triangles), and C32V $+\mathrm{V}$ (open circles), respectively. C32V $+\mathrm{C}$ exhibits a positive linear correlation between $E$ and $R_{c}$, and the slope of dot line is 31.6. In the case of $\mathrm{C} 32 \mathrm{~V}+\mathrm{V}$, no correlation is observed.

Ex436 and Ex470 or Ex436 and Ex480 excitations should be good choices for quantitative mExEm-spFRET measurement of $\mathrm{CFP} /$ Cerulean and YFP/Venus pairs. In reality, we can perform a quantitative mExEm-spFRET measurement with two excitations within $1 \mathrm{~s}$, which is applicable to the monitoring of dynamical events in single living cells.

\section{5 mExEm-spFRET Measurement of C32V in the Presence of Free Donor or Free Acceptor}

We also used mExEm-spFRET method with four excitation wavelengths to measure the $E$ and $R_{c}$ values of $\mathrm{C} 32 \mathrm{~V}$ construct in the presence of free Cerulean or free Venus. Figure 5 shows the $E-R_{c}$ plot on a cell-by-cell basis for $\mathrm{C}+\mathrm{V}, \mathrm{C} 32 \mathrm{~V}, \mathrm{C} 32 \mathrm{~V}+$ $\mathrm{C}$, and $\mathrm{C} 32 \mathrm{~V}+\mathrm{V}$, respectively. Unlinked Cerulean plus Venus $(\mathrm{C}+\mathrm{V})$ exhibits very low $E$ values independent of $R_{c}$ (solid squares), whereas $\mathrm{C} 32 \mathrm{~V}$ exhibits a restricted distribution for $E$ (about $30.9 \%$ ) and $R_{c}$ (about 1.02) values (solid triangles). The $E$ values of $\mathrm{C} 32 \mathrm{~V}+\mathrm{C}$ are positively proportional to the corresponding $R_{c}$ (open triangles), whereas $\mathrm{C} 32 \mathrm{~V}+\mathrm{V}$ has

Table $2 E$ and $R_{C}$ values of constructs measured by mExEm-spFRET with different excitation wavelengths.

\begin{tabular}{|c|c|c|c|c|c|c|c|c|}
\hline \multirow[b]{3}{*}{ Excitation wavelengths (nm) } & \multicolumn{8}{|c|}{ Constructs } \\
\hline & \multicolumn{2}{|c|}{$C+V$} & \multicolumn{2}{|c|}{$\mathrm{C} 32 \mathrm{~V}$} & \multicolumn{2}{|c|}{ CVC } & \multicolumn{2}{|c|}{ VCV } \\
\hline & $E(\%)$ & $R_{c}$ & $E(\%)$ & $R_{c}$ & $E(\%)$ & $R_{c}$ & $E(\%)$ & $R_{c}$ \\
\hline $405,436,470,480$ & $3.1 \pm 1.5$ & $1.00 \pm 0.33$ & $30.9 \pm 2.0$ & $1.02 \pm 0.04$ & $40.5 \pm 2.3$ & $0.51 \pm 0.02$ & $69.6 \pm 1.6$ & $2.37 \pm 0.11$ \\
\hline 405,436 & $2.4 \pm 1.6$ & $0.92 \pm 0.58$ & $29.6 \pm 2.2$ & $1.00 \pm 0.11$ & $38.7 \pm 2.4$ & $0.70 \pm 0.11^{*}$ & $68.3 \pm 1.7$ & $2.49 \pm 0.23$ \\
\hline 405,470 & $3.6 \pm 1.7$ & $1.08 \pm 0.72$ & $31.6 \pm 2.0$ & $0.99 \pm 0.04$ & $40.7 \pm 2.3$ & $0.54 \pm 0.03$ & $70.3 \pm 1.6$ & $2.45 \pm 0.12$ \\
\hline 405,480 & $2.8 \pm 1.5$ & $1.08 \pm 0.71$ & $30.6 \pm 2.1$ & $1.01 \pm 0.03$ & $39.9 \pm 2.3$ & $0.51 \pm 0.03$ & $69.6 \pm 1.6$ & $2.41 \pm 0.11$ \\
\hline 436,470 & $3.1 \pm 1.5$ & $1.10 \pm 0.71$ & $31.0 \pm 1.9$ & $0.99 \pm 0.05$ & $40.1 \pm 2.2$ & $0.53 \pm 0.03$ & $69.4 \pm 1.6$ & $2.42 \pm 0.11$ \\
\hline 436,480 & $2.9 \pm 1.5$ & $1.07 \pm 0.69$ & $30.7 \pm 1.9$ & $0.97 \pm 0.04$ & $40.0 \pm 2.2$ & $0.50 \pm 0.02$ & $69.8 \pm 1.6$ & $2.31 \pm 0.10$ \\
\hline
\end{tabular}

${ }^{\star} P<0.05$, compared with the corresponding $R_{c}$ value with four excitation wavelengths (the first line). 
(a)

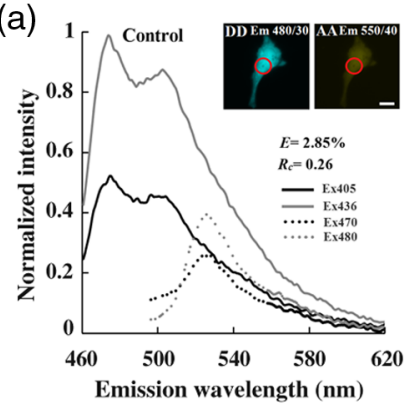

(b)

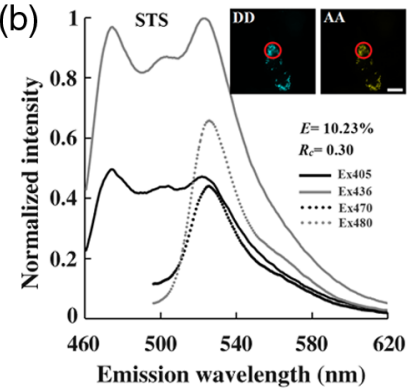

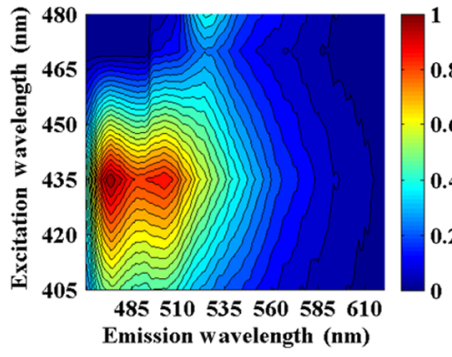

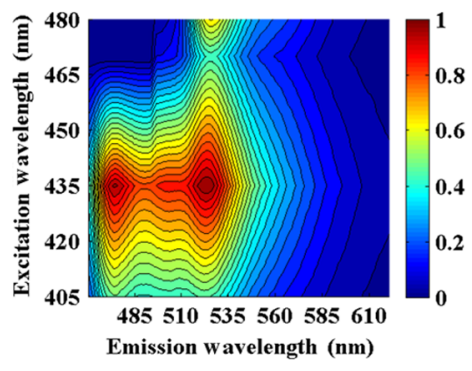

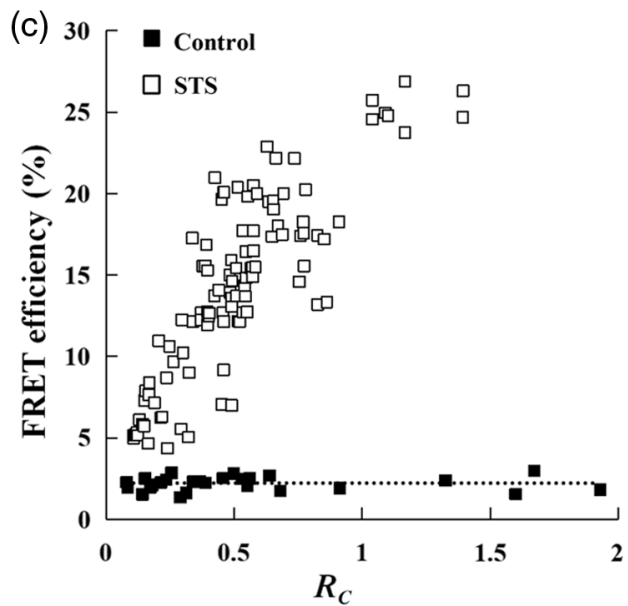

Fig. 6 mExEm-spFRET analysis on Bax homooligomerization in single living HeLa cells coexpressing CFP-Bax and YFP-Bax. (a and b) Normalized emission spectra (left) and the corresponding excitationemission spectrum (right) of a representative cell coexpressing CFP-Bax and YFP-Bax in the absence (a) and presence (b) of STS with Ex405, Ex436, Ex470, and Ex480 excitation, respectively, with respect to the value of the maximum peak at emission spectrum with Ex436 excitation. Scale bar: $10 \mu \mathrm{m}$. (c) $E-R_{c}$ plot from 26 control cells (solid squares) and 105 STS-treated cells (open squares), respectively.

the same $E$ values as $\mathrm{C} 32 \mathrm{~V}$ (open circles), which is consistent with the previous reports. ${ }^{15,20,34}$

In reality, high concentration of free Cerulean and free Venus may result in the possibility of spurious FRET efficiency by random collision. ${ }^{35}$ For some bright cells coexpressing Cerulean and Venus $(\mathrm{C}+\mathrm{V}$, solid squares in Fig. 5), donor and acceptor may be within the Förster distance and form "spurious donoracceptor complex," which leads to a small systematic increase of $E$ as a function of $R_{c} \cdot{ }^{10,14}$ Therefore, we should not choose the cells with very high concentration of fluorescent proteins for quantitative measurements.

\section{6 mExEm-spFRET Measurement of STS-Induced Bax Homeoligomerization}

Bax is a proapoptotic protein required for the process of mitochondrial outer membrane permeabilization. ${ }^{1}$ Some publications, including our previous studies, have demonstrated that STS induces Bax translocation into mitochondria and subsequent homooligomerization..$^{20,28,36}$ We here performed mExEm-spFRET method on SM for single living HeLa cells coexpressing CFP-Bax and YFP-Bax. A CFP-YFP tandem reference $(18 \mathrm{AA})$ was used to predetermine the $f_{\mathrm{sc}}(1.80)$ and $r_{k}(0.42)$ values for CFP-YFP pair on our SM system. As shown in Figs. 6(a) and 6(b), emission spectrum with Ex405, Ex436, Ex470, and Ex480 excitation, respectively, was divided by the maximum value of emission spectrum with Ex436 excitation to obtain the normalized emission spectra. Bax distributed evenly in cytosol in the control cell exhibiting $2.85 \%$ of $E$ and 0.26 of $R_{c}$ [Fig. 6(a)], and Bax showed significant clusters in the cell exhibiting $10.23 \%$ of $E$ and 0.30 of $R_{c}$ after $2-\mu \mathrm{M}$ STS treatment for $6 \mathrm{~h}$ [Fig. 6(b)]. Statistical $E$ values are $2.2 \% \pm 0.4 \%$ for control cells (26 cells) and $14.4 \% \pm$ $5.7 \%$ for STS-treated cells (105 cells), indicating that STS induced the formation of mitochondria-associated Bax clusters. Figure 6(c) shows the $E-R_{c}$ plot on a cell-by-cell basis for control (solid squares) and STS-treated (open squares) cells, respectively. As shown in Fig. 6(c), the fact that the FRET efficiency \{apparent FRET efficiency $\left[E_{\mathrm{app}}=E_{\max } \cdot(D A) /\right.$ $\left.\left.\left(D_{\text {total }}\right)\right]\right\}$ is very low and independent on the $R_{c}$ for control cells indicates that Bax does not exist as homooligomer in healthy cells. However, for the STS-treated cells, apparent FRET efficiency $\left(E_{\text {app }}\right)$ obviously increased in the case of $R_{c}<1\left[(D A)\right.$ increases with $R_{c}$ or $\left.\left(A_{\text {total }}\right)\right]$ but kept constant in the case of $R_{c}>1\left[(D A)=\left(D_{\text {total }}\right)\right]$ with $R_{c}$ increasing, further demonstrating that all Bax formed homooligomer after STS treatment for $6 \mathrm{~h}$.

\section{Conclusions}

We here set up an improved SM for fast quantitative ExEmspFRET measurement in single living cells. Our SM system is very stable for at least six months. The modified ExEm-spFRET method (mExEm-spFRET) containing a system correction factor $\left(f_{\mathrm{sc}}\right)$ can be easily performed on our SM platform for quantitative FRET measurement in single living cells. Especially, availability of mExEm-spFRET with two excitation wavelengths enables the SM system to implement real-time and dynamical mExEm-spFRET measurement in single living cells, which is very important for monitoring intracellular rapid biochemical events.

\section{Appendix: Emission Spectral Responses of SM System}

As shown in Fig. 7, we carefully measured the emission spectral responses $[K(\lambda)]$ of SM system. $E(\lambda)$ and count ${ }_{\text {lamp }}(\lambda)$ were normalized at emission wavelength $620 \mathrm{~nm}$. 

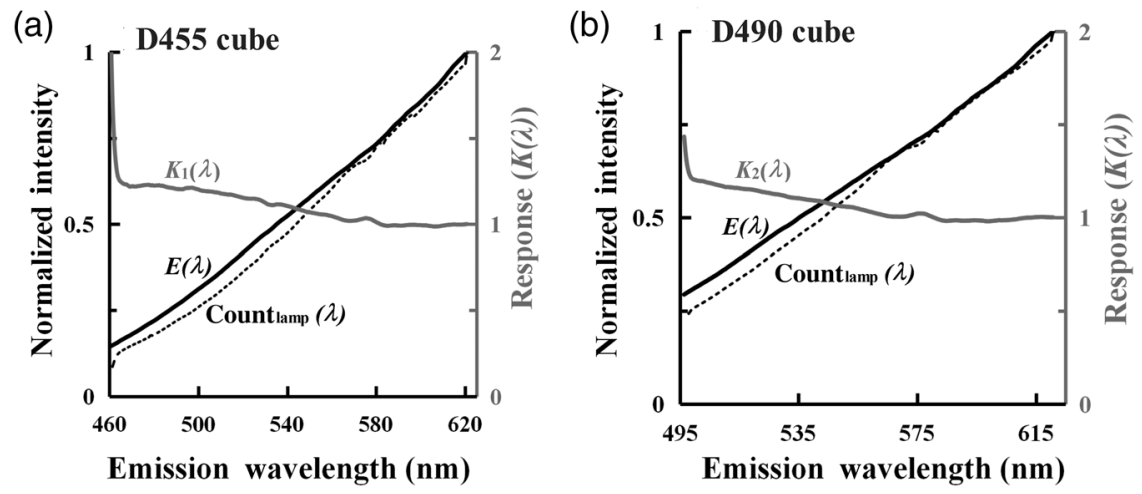

Fig. 7 Emission spectral responses of SM system. (a) Spectrum of halogen tungsten lamp $[E(\lambda)$, black solid line] measured using the spectrometer. Spectrum measured from the export of our microscope with D455 cube containing a dichroic filter D455 and a long-pass filter LP460 [count $t_{\text {lamp }}(\lambda)$, dot line] when the halogen tungsten lamp was placed on the objective. Spectral response curve of SM system with D455 cube $\left[K_{1}(\lambda)\right.$, gray solid line]. $E(\lambda)$ and count lamp $(\lambda)$ are normalized at emission wavelength $\lambda=620 \mathrm{~nm}$. (b) Same as (a), except that spectrum [count lamp $(\lambda)$, dot line] measured from the export of our microscope with D490 cube containing a dichroic filter D490 and a long-pass filter LP495.

\section{Disclosures}

The authors have no competing interests.

\section{Acknowledgments}

We thank Professor S.S. Vogel (NIH/NIAAA) for providing $\mathrm{C} 32 \mathrm{~V}, \mathrm{CVC}$, and VCV plasmids, Dr. Prehn for providing CFP-Bax and YFP-Bax plasmids, and Professor Kaminski for providing 18AA plasmid. This project was supported by the National Natural Science Foundation of China (Grant Nos. 81471699 and 61527825) and the Science and Technology Plan Project of Guangdong Province (No. 2014B090901060).

\section{References}

1. H. Düssmann et al., "Single-cell quantification of Bax activation and mathematical modeling suggest pore formation on minimal mitochondrial Bax accumulation," Cell Death Differ. 17(2), 278-290 (2010).

2. R. F. Gahl et al., "Conformational rearrangements in the pro-apoptotic protein, Bax, as it inserts into mitochondria: a cellular death switch," J. Biol. Chem. 289(47), 32871-32882 (2014).

3. R. Onuki et al., "Confirmation by FRET in individual living cells of the absence of significant amyloid $\beta$-mediated caspase 8 activation," Proc. Natl. Acad. Sci. U. S. A. 99(23), 14716-14721 (2002).

4. F. Wang et al., "Measuring dynamics of caspase-3 activity in living cells using FRET technique during apoptosis induced by high fluence low-power laser irradiation," Lasers Surg. Med. 36(1), $2-7$ (2005).

5. Y. Nagai et al., "A fluorescent indicator for visualizing cAMP-induced phosphorylation in vivo," Nat. Biotechnol. 18(3), 313-316 (2000).

6. K. Kurokawa et al., "A pair of fluorescent resonance energy transferbased probes for tyrosine phosphorylation of the CrkII adaptor protein in vivo," J. Biol. Chem. 276(33), 31305-31310 (2001).

7. A. Miyawaki et al., "Fluorescent indicators for $\mathrm{Ca}^{2+}$ based on green fluorescent proteins and calmodulin," Nature 388(6645), 882-887 (1997).

8. A. Woehler, "Simultaneous quantitative live cell imaging of multiple FRET-based biosensors," PLoS One 8(4), e61096 (2013).

9. A. D. Hoppe, K. Christensen, and J. A. Swanson, "Fluorescence resonance energy transfer-based stoichiometry in living cells," Biophys. J. 83(6), 3652-3664 (2002).

10. T. Zal and N. R. J. Gascoigne, "Photobleaching-corrected FRET efficiency imaging of live cells," Biophys. J. 86(6), 3923-3939 (2004).

11. A. D. Elder et al., "A quantitative protocol for dynamic measurements of protein interactions by Forster resonance energy transfer-sensitized fluorescence emission," J. R. Soc. Interface 6(Suppl. 1), S59-S81 (2009).
12. T. Förster, "Excitation transfer, intermolecular energy transference and fluorescence," Ann. Phys. 437(2), 55-75 (1948).

13. T. Zimmermann, "Spectral imaging and linear unmixing in light microscopy," Adv. Biochem. Eng. 95, 245-265 (2005).

14. J. Wlodarczyk et al., "Analysis of FRET signals in the presence of free donors and acceptors," Biophys. J. 94(3), 986-1000 (2008).

15. S. Levy et al., "SpRET: highly sensitive and reliable spectral measurement of absolute FRET efficiency," Microsc. Microanal. 17(2), 176190 (2011).

16. S. Mustafa et al., "Quantitative Förster resonance energy transfer efficiency measurements using simultaneous spectral unmixing of excitation and emission spectra," J. Biomed. Opt. 18(2), 026024 (2013).

17. A. D. Hoppe et al., "N-way FRET microscopy of multiple proteinprotein interactions in live cells," PLoS One 8(6), e64760 (2013).

18. R. M. Clegg, "Fluorescence resonance energy transfer and nucleic acids," Methods Enzymol. 211, 353-388 (1992).

19. C. Thaler et al., "Quantitative multiphoton spectral imaging and its use for measuring resonance energy transfer," Biophys. J. 89(4), 2736-2749 (2005).

20. J. W. Zhang et al., "Quantitative FRET measurement using emissionspectral unmixing with independent excitation crosstalk correction," J. Microsc. 257(2), 104-116 (2015).

21. Y. Chen et al., "Characterization of spectral FRET imaging microscopy for monitoring nuclear protein interactions," J. Microsc. 228(2), 139_ 152 (2007).

22. T. Zimmermann, J. Rietdorf, and R. Pepperkok, "Spectral imaging and its applications in live cell microscopy," FEBS Lett. 546(1), 87-92 (2003).

23. J. Yuan et al., "Quantitative FRET measurement by high-speed fluorescence excitation and emission spectrometer," Opt. Express 18(18), 18839-18851 (2010).

24. L. Y. Chai et al., "Miniature fiber optic spectrometer-based quantitative fluorescence resonance energy transfer measurement in single living cells," J. Biomed. Opt. 20(3), 037008 (2015).

25. M. Y. Du et al., "Wide-field microscopic FRET imaging using simultaneous spectral unmixing of excitation and emission spectra," Opt. Express 24(14), 16037-16051 (2016).

26. H. N. Yu et al., "An empirical quantitative fluorescence resonance energy transfer method for multiple acceptors based on partial acceptor photobleaching," Appl. Phys. Lett. 100(25), 253701 (2012).

27. S. V. Koushik, P. S. Blank, and S. S. Vogel, "Anomalous surplus energy transfer observed with multiple FRET acceptors," PLoS One 4(11), e8031 (2009)

28. J. Zhang et al., "IIem-spFRET: improved Iem-spFRET method for robust FRET measurement," J. Biomed. Opt. 21(10), 105003 (2016).

29. R. N. Day and M. W. Davidson, "Fluorescent proteins for FRET microscopy: monitoring protein interactions in living cells," Bioessays 34(5), 341-350 (2012) 
30. H. W. Ai et al., "Directed evolution of a monomeric, bright and photostable version of Clavularia cyan fluorescent protein: structural characterization and applications in fluorescence imaging," Biochem. J. 400(3), 531-540 (2006).

31. M. A. Rizzo et al., "An improved cyan fluorescent protein variant useful for FRET," Nat. Biotechnol. 22(4), 445-449 (2004).

32. T. Nagai et al., "A variant of yellow fluorescent protein with fast and efficient maturation for cell-biological applications," Nat. Biotechnol. 20(7), 87-90 (2002).

33. H. Chen et al., "Measurement of FRET efficiency and ratio of donor to acceptor concentration in living cells," Biophys. J. 91(5), L39-L41 (2006).

34. L. L. Zhang et al., "Spectral wide-field microscopic fluorescence resonance energy transfer imaging in live cells," J. Biomed. Opt. 20(8), 086011 (2015).

35. E. S. Butz et al., "Quantifying macromolecular interactions in living cells using FRET two-hybrid assays," Nat. Protoc. 11(12), 2470-2498 (2016).

36. S. S. Smaili et al., "Bax translocation to mitochondria subsequent to a rapid loss of mitochondrial membrane potential," Cell Death Differ. 8(9), 909-920 (2001).
Fangrui Lin is pursuing his MS degree at South China Normal University. His current research interests include fluorescence microscopy and biomedical optics.

Mengyan Du is pursuing her MS degree at South China Normal University. Her current research interests include quantitative fluorescence resonance energy transfer (FRET) imaging.

Fangfang Yang is pursuing her doctoral degree at South China Normal University. Her current research interests include quantitative FRET imaging and cell biology.

Lichun Wei is pursuing her MS degree at South China Normal University. Her current research interests include fluorescence microscopy and biomedical optics.

Tongsheng Chen received his $\mathrm{PhD}$ in biomedical engineering from Huazhong University of Science and Technology, China. He has 19 years of experience in fluorescence microscopy and biomedical optics. He is the author of more than 120 journal papers and also a member of SPIE. 